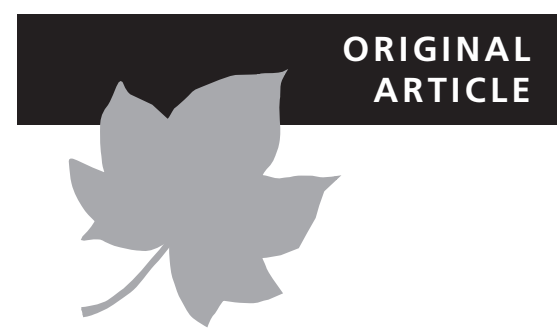

\title{
Partitioning taxon, phylogenetic and functional beta diversity into replacement and richness difference components
}

Pedro Cardoso ${ }^{1,2 \star}$, François Rigal ${ }^{2}$, José C. Carvalho ${ }^{2,3}$, Mikael Fortelius ${ }^{1,4}$, Paulo A. V. Borges ${ }^{2}$, Janos Podani ${ }^{5,6}$ and Denes Schmera ${ }^{7,8}$

${ }^{1}$ Finnish Museum of Natural History, University of Helsinki, Helsinki, Finland, ${ }^{2}$ Azorean Biodiversity Group (CITA-A) and Portuguese Platform for Enhancing Ecological Research \& Sustainability (PEERS), University of the Azores, Angra do Heroísmo, Portugal, ${ }^{3}$ Department of Biology, CBMA Molecular and Environmental Centre, University of Minho, Braga, Portugal, ${ }^{4}$ Department of Geosciences and Geography, University of Helsinki, Helsinki, Finland, ${ }^{5}$ Department of Plant Systematics, Ecology and Theoretical Biology, Institute of Biology, Eötvös University, Budapest, Hungary, ${ }^{6}$ Ecology Research Group, Hungarian Academy of Sciences, Budapest, Hungary, ${ }^{7}$ Section of Conservation Biology, University of Basel, Basel, Switzerland, ${ }^{8}$ Balaton Limnological Institute, Centre for Ecological Research, Hungarian Academy of Sciences, Klebelsberg K. u. 3, Tihany, Hungary

${ }^{*}$ Correspondence: Pedro Cardoso, Finnish Museum of Natural History, P.O. Box 17, 00014 Helsinki, Finland.

E-mail: pedro.cardoso@helsinki.fi

\begin{abstract}
Aim To propose a unified framework for quantifying taxon (T $\beta$ ), phylogenetic $(\mathrm{P} \beta)$ and functional $(\mathrm{F} \beta)$ beta diversity via pairwise comparisons of communities, which allows these types of beta diversity to be partitioned into ecologically meaningful additive components.
\end{abstract}

Location Global, with case studies in Europe and the Azores archipelago.

Methods Using trees as a common representation for taxon, phylogenetic and functional diversity, we partition total beta diversity $\left(\beta_{\text {total }}\right)$ into its replacement (turnover, $\left.\beta_{\text {repl }}\right)$ and richness difference $\left(\beta_{\text {rich }}\right)$ components according to which part of a global tree was shared by or unique to communities that were being compared. We demonstrate the application of this framework using artificial and empirical examples (mammals in Europe and epigean arthropods in the Azores).

Results Our empirical examples show that comparing $\mathrm{P} \beta$ and $\mathrm{F} \beta$ with the most commonly used $\mathrm{T} \beta$ revealed previously hidden patterns of beta diversity. More importantly, we demonstrate that partitioning $P \beta_{\text {total }}$ and $F \beta_{\text {total }}$ into their respective $\beta_{\text {repl }}$ and $\beta_{\text {rich }}$ components facilitates the detection of more complex patterns than using the overall coefficients alone, further elucidating the different forces operating in community assembly.

Main conclusions The methods presented here allow the integration and full comparison of $\mathrm{T} \beta, \mathrm{P} \beta$ and $\mathrm{F} \beta$. They provide a tool for effectively disentangling the replacement (turnover) and richness difference components of the different biodiversity facets within the same methodological framework.

\section{Keywords}

Azores, arthropods, beta diversity metrics, differentiation, dissimilarity, European mammals, functional diversity, phylogenetic diversity, taxonomic diversity, turnover.

\section{INTRODUCTION}

Assessing, measuring and interpreting biodiversity in space and time are challenging tasks in ecology, evolution, biogeography and conservation. The notion of taxon diversity (TD) is the most common and is quantified in many different ways based on the number of taxa and, often, on the distribution of abundances. TD treats taxa as being equally distinct from one another and disregards the fact that communities are composed of species with different evolutionary histories (Webb et al., 2002; Graham \& Fine, 2008) and a diverse array of ecological functions (Villéger

(c) 2013 John Wiley \& Sons Ltd et al., 2012, 2013). Thus, the last decade has seen a growing interest in alternative representations of biodiversity, including phylogenetic diversity (PD) and functional diversity (FD) (Devictor et al., 2010; Meynard et al., 2011; Stegen \& Hurlbert, 2011). Although TD, PD and FD may be correlated, they can reveal different mechanisms associated with the origin and maintenance of biodiversity. Note that although the terms 'phylogenetic diversity' and 'functional diversity' have been used to refer to a specific class of measures, we use them as generic terms for any method for integrating phylogenetic or functional information into diversity measures. 
Phylogenetic diversity takes the evolutionary relationships between taxa into account (Faith, 1992) and reflects how much evolutionary history is behind the species in the community. Communities with identical TD may differ widely with respect to their evolutionary past, depending on how far the species have diverged from their nearest common ancestor (Webb et al., 2002; Graham \& Fine, 2008). PD has mostly been measured based on phylogenetic trees or cladograms. The available measures encompass either the amount of phylogenetic information conveyed by the community - e.g. the minimum length of tree edges connecting all taxa (Faith, 1992) - or the degree of (un)relatedness of taxa - e.g. the average node distance between them in the phylogenetic tree (Webb et al., 2002; but see Helmus et al., 2007, for alternative measures).

In lieu of precise phylogenetic information on the member taxa of a community, taxonomic diversity (LD, the abbreviation referring to Linnaeus) may be used as a surrogate for $\mathrm{PD}$, and may be calculated in a similar way from an ultrametric tree (i.e. a dissimilarity matrix) representing the Linnaean hierarchy (Ricotta et al., 2012). Consequently, LD uses the hierarchical classification of organisms, whereas TD does not, and it approximates the amount of information provided by PD with variable success depending on how closely the Linnaean system represents the phylogeny as hypothesized by the phylogenetic tree. Thus, 'taxon' and 'taxonomic' diversity are not the same; the first refers to a partition, whereas the second refers to a hierarchy.

Functional diversity quantifies the components of biodiversity that influence how an ecosystem operates or functions (Tilman et al., 2001). Communities with completely different species composition may be characterized by low variation in functional traits, with unrelated species replacing others with similar roles in the network (Villéger et al., 2012, 2013). FD has also been quantified in many different ways, often using approaches that were first developed for $\mathrm{PD}$, such as quadratic entropy (Rao, 1982), dendrogram-based measures (Petchey \& Gaston, 2002, 2006) or the functional hyperspace occupied by taxa (Villéger et al., 2013).

Although $\beta$-diversity has been most frequently studied at the taxon level (i.e. T $\beta$ ), as originally suggested (Whittaker, 1960; Anderson et al., 2011), there is growing demand to understand the phylogenetic and functional components of community variation as well (see Swenson, 2011, and references therein). This inevitably leads to $\beta$ being defined in terms of the phylogenetic or functional relatedness of member species of a given community. Phylogenetic beta diversity $(\mathrm{P} \beta)$ allows one to discriminate phylogenetically basal and terminal changes (branch points occurring early and late in a phylogeny) between communities, a feature that is impossible to reveal using taxon-based analyses (Graham \& Fine, 2008). Functional beta diversity (F $\beta$ ) intends to discriminate change between communities of taxa with either similar or different functions in the system (see, for example, Ricotta \& Burrascano, 2008).

Many $\beta$ measures proposed to date reduce the calculations to pairwise comparisons of communities. Examples are the two most commonly used P $\beta$ measures: UniFrac (a phylogenetic equivalent of the Jaccard index; Lozupone \& Knight, 2005) and PhyloSor (a phylogenetic equivalent of the Sørensen index; Bryant et al., 2008) (see also Rao's quadratic entropy as a common measure for $\mathrm{T} \beta, \mathrm{P} \beta$ and $\mathrm{F} \beta$; Devictor et al., 2010; but see Chao et al., 2010, for problems using Rao's entropy because of nonlinearity with increasing diversity). These indices are applied to tree representations of communities: a phylogenetic tree is obtained for the member species, and the coefficients are calculated from the matching and mismatching edges of the tree. Each coefficient has different (dis)advantages and interpretations (e.g. Swenson, 2011; Feng et al., 2012); it is difficult to infer process from pattern because the coefficients do not provide information about exact drivers. Therefore, compositional differences driven by true replacement of lineages are not distinguishable from differences that are driven by the loss (or gain) of entire lineages (Leprieur et al., 2012). The same could be said of all $\mathrm{F} \beta$ measures, because they do not separate compositional changes due to true replacement of functional traits from differences due to the loss (or gain) of traits (Villéger et al., 2013).

A solution is offered by beta-diversity partitioning, i.e. separating components that originate from different underlying processes (not to be confounded with gamma-diversity partitioning into alpha and beta components; Lande, 1996; Veech et al., 2002; Jost, 2007). Pioneering work in beta diversity partitioning was conducted by Baselga (2010), who suggested partitioning $\mathrm{T} \beta$ into turnover and nestednessresultant dissimilarity fractions, as derived from both the Sørensen and Jaccard indices (Baselga, 2010, 2012). These approaches were adapted to the analysis of $\mathrm{P} \beta$ (Leprieur et al., 2012) and F $\beta$ (Villéger et al., 2013), but such methods present several shortcomings (see below; Schmera \& Podani, 2011; Almeida-Neto et al., 2012; Carvalho et al., 2012, 2013). We have previously demonstrated how $\mathrm{T} \beta$ should be disentangled into algebraically comparable fractions, reflecting the replacement and loss (or gain) of taxa in an ecologically meaningful manner (Schmera \& Podani, 2011; Carvalho et al., 2012, 2013). It has been shown that partitioning total T $\beta$ into such components has significantly improved our understanding of community organization in space and time (Baiser et al., 2012; Marini et al., 2013).

Our objective is to extend this taxon-based approach to phylogenetic and functional analyses. This unified framework allows meaningful comparisons of all aspects of beta diversity $(\mathrm{T} \beta, \mathrm{P} \beta$ and $\mathrm{F} \beta$ ) and their replacement and richness difference components. We demonstrate its application using theoretical and empirical data sets.

\section{A UNIFIED FRAMEWORK FOR TAXON, PHYLOGENETIC AND FUNCTIONAL BETA DIVERSITY}

The proposed framework uses a global tree $G=(V, E)$ that is derived from phylogenetic or functional data for the 
members of all the communities of interest. $G$ comprises a set $V$ of nodes or vertices and a set $E$ of lines or edges. The terminal vertices in $G$ correspond to taxa (often species), whereas interior nodes represent either sister-group relationships in cladograms or fusion steps in hierarchical classifications. For each community, we select those edges that connect the terminals (taxa) to the root of the global tree. Hence, for each community $j$, we obtain a community tree $G_{j}=\left(V_{j}, E_{j}\right)$, which is a subtree of the global tree. Note that taxon diversity (TD) can also be visualized using a tree diagram, although it is a very special one: in its global tree, each taxon is linked directly to the root by an edge of unit length (star tree). Thus, tree diagrams provide a common basis for an unequivocal comparison of TD, PD (or its surrogate, LD) and FD. Using this general framework, $\alpha$-diversity $(\mathrm{T} \alpha, \mathrm{P} \alpha$ or $\mathrm{F} \alpha$ ) for a given community $j$ is given by:

$$
\alpha_{j}=\sum_{e_{x} \in E_{j}} l_{x}
$$

where $l_{x}$ represents the length $(l)$ of edge $e_{x}$ in $G_{j}$. Thus, $\alpha_{j}$ is the length of tree $G_{j}$.

Total dissimilarity, understood here as the total pairwise $\beta$ of communities $j$ and $k$, corresponds to the sum of the lengths of edges that are unique to each community tree:

$$
\text { total dissimilarity }{ }_{j k}=\sum_{e_{x} \in E_{j}} l_{x}+\sum_{e_{x} \in E_{k}} l_{x}-2 \sum_{e_{x} \in E_{j} \cap E_{k}} l_{x} .
$$

This equation was implicitly or explicitly part of previous methods describing total phylogenetic dissimilarity (UniFrac, Lozupone \& Knight, 2005; PhyloSor, Bryant et al., 2008). Our basic idea is that total dissimilarity can be partitioned additively into two fractions - replacement and richness difference. The first one corresponds to the substitution of edges exclusive to community $j$ by other edges with the same total length that are exclusive to $k$. Algebraically, the replacement fraction of the total dissimilarity is given by twice the minimum of the sum of the lengths of edges that are unique to each community:

replacement $_{j k}=2 \min \left\{\sum_{e_{x} \in E_{j} \cup E_{k}} l_{x}-\sum_{e_{x} \in E_{j}} l_{x}, \sum_{e_{x} \in E_{j} \cup E_{k}} l_{x}-\sum_{e_{x} \in E_{k}} l_{x}\right\}$.

The remaining dissimilarity fraction corresponds to difference in $\alpha$-diversity or richness, and is quantified by the absolute difference between the lengths of $G_{j}$ and $G_{k}$ :

$$
\text { richness difference }{ }_{j k}=\left|\sum_{e_{x} \in E_{j}} l_{x}-\sum_{e_{x} \in E_{k}} l_{x}\right|=\left|\alpha_{j}-\alpha_{k}\right| .
$$

In fact, the total dissimilarity measure is the algebraic sum of the replacement and richness difference measures. Finally, all these quantities must be scaled in relation to a common denominator. This should be the sum of the lengths of edges that are part of either community $j$ or $k$, including shared edges, i.e. gamma diversity in a pairwise comparison:

$$
\gamma_{j k}=\sum_{e_{x} \in E_{j} \cup E_{k}} l_{x} .
$$

From a different, complementary perspective, pairwise beta diversity has traditionally been measured by comparing the matching/mismatching components of a $2 \times 2$ contingency table (Nipperess et al., 2010; Legendre \& Legendre, 2012). These components are the number of features (a) common to both communities, (b) present in the first community only, (c) present in the second community only, and (d) absent from both communities but present elsewhere in the region of interest. Such features are usually species. For tree representations, the features of interest are not species but edges, which may have different lengths and be shared by different species which may be present in different communities (Fig. 1). Following Nipperess et al. (2010), the matching component $a$ is the sum of the lengths of the edges pertaining to taxa shared by both communities $j$ and $k$ :

$$
a=\sum_{e_{x} \in E_{j} \cap E_{k}} l_{x}
$$

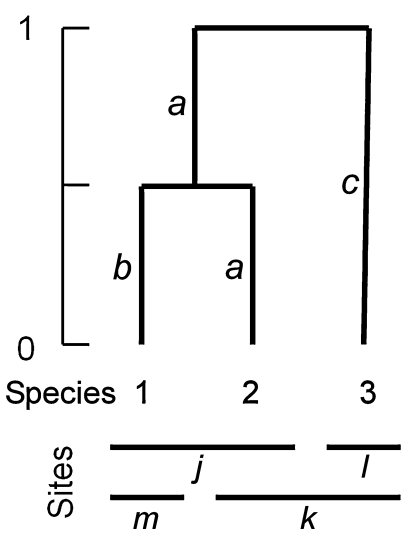

Figure 1 Theoretical example of a phylogenetic or functional tree, with three species and four communities. The matching (a) and mismatching $(b, c)$ components between two communities $(j, k)$ are also shown. Comparison of $j$ and $k$ is an example of species replacement without richness difference in a taxon-based analysis because each has two species. Because $k$ has a longer exclusive edge adjacent to species 3 than $j$ has to species 1 , part of total phylogenetic beta diversity $\left(\mathrm{P} \beta_{\text {total }}\right)$ or total functional beta diversity $\left(\mathrm{F} \beta_{\text {total }}\right)$ is due to richness difference (Table 1$)$. Communities $j$ and $l$ have no species or edges in common. However, edges connecting the species of $j$ to the root are longer than the single edge in $l$ (1.5 vs. 1 unit), and this is reflected in the richness difference fraction. Communities $j$ and $m$ or $k$ and $l$ illustrate cases of pure loss of species and associated edges with no replacement. Communities $k$ and $m$ have no species in common, but share one edge; hence, $\mathrm{P} \beta_{\text {total }}$ or $\mathrm{F} \beta_{\text {total }}$ is lower than 1 . Communities $l$ and $m$ share neither species nor edges, and both have the same alpha diversity, leading to maximum replacement and no richness difference. 
The mismatching component $b$ is the sum of the lengths of the edges pertaining to taxa present in $j$ but not in $k$ :

$$
b=\sum_{e_{x} \in E_{j}} l_{x}-\sum_{e_{x} \in E_{j} \cap E_{k}} l_{x}=\alpha_{j}-a .
$$

Analogously, the mismatching component $c$ is the sum of the lengths of the edges pertaining to taxa present in $k$ but not in $j$ :

$$
c=\sum_{e_{x} \in E_{k}} l_{x}-\sum_{e_{x} \in E_{j} \cap E_{k}} l_{x}=\alpha_{k}-a .
$$

Therefore, $\gamma_{j k}=a+b+c$. The parameter $d$ is not used in beta diversity measures, because double zeros are usually considered uninformative (Legendre \& Legendre, 2012). Based on these matching/mismatching components, it is now possible to formally link our new measures to previous approaches for partitioning pairwise T $\beta$ (Podani \& Schmera, 2011; Carvalho et al., 2012). $\beta_{\text {total }}$ is therefore

$$
\beta_{\text {total }}=\frac{\sum_{e_{x} \in E_{j}} l_{x}+\sum_{e_{x} \in E_{k}} l_{x}-2 \sum_{e_{x} \in E_{j} \cap E_{k}} l_{x}}{\sum_{e_{x} \in E_{j} \cup E_{k}} l_{x}}=\frac{b+c}{a+b+c} .
$$

When applied to TD, $\beta_{\text {total }}$ is equivalent to pairwise beta diversity as expressed by the Jaccard index of dissimilarity. For quantitative data, such as edge lengths, Jaccard dissimilarity is in fact a generalization to the Marczewski-Steinhaus coefficient of dissimilarity that is often used in multivariate analysis (Podani et al., 2013). This measure is better known as UniFrac (Lozupone \& Knight, 2005) in PD studies.

Relativized beta due to replacement or turnover $\left(\beta_{\text {repl }}\right)$ is as follows:

$$
\begin{aligned}
\beta_{\text {repl }} & =\frac{2 \min \left\{\sum_{e_{x} \in E_{j} \cup E_{k}} l_{x}-\sum_{e_{x} \in E_{j}} l_{x}, \sum_{e_{x} \in E_{j} \cup E_{k}} l_{x}-\sum_{e_{x} \in E_{k}} l_{x}\right\}}{\sum_{e_{x} \in E_{j} \cup E_{k}} l_{x}} \\
& =\frac{2 \min \{b, c\}}{a+b+c} .
\end{aligned}
$$

When applied to TD, $\beta_{\text {repl }}$ is equivalent to beta diversity derived from the Williams $\beta_{-3}$ measure, as modified by Cardoso et al. (2009).

Finally, relativized pairwise beta due to richness difference among communities $\left(\beta_{\text {rich }}\right)$ is as follows:

$$
\beta_{\text {rich }}=\frac{\left|\sum_{e_{x} \in E_{j}} l_{x}-\sum_{e_{x} \in E_{k}} l_{x}\right|}{\sum_{e_{x} \in E_{j} \cup E_{k}} l_{x}}=\frac{|b-c|}{a+b+c} .
$$

When applied to TD, $\beta_{\text {rich }}$ is equivalent to the beta diversity derived from species richness difference, as defined by Podani \& Schmera (2011) and Carvalho et al. (2012). Note that a similar formula, derived from the Sørensen index, was proposed by Lennon et al. (2001). Because the denominator is identical in the above three equations, we may derive the following:

$$
\beta_{\text {total }}=\beta_{\text {repl }}+\beta_{\text {rich }}
$$

\section{THEORETICAL CASES}

\section{A small theoretical dataset}

To better understand the application of the novel framework, we first use a theoretical representation of either a phylogenetic or a functional global tree for a given sampled universe (Fig. 1, Table 1). All measures behave in an intuitive way, correctly reflecting the different proportions of replacement and richness difference.

\section{A large theoretical dataset}

The behaviour of the new measures was also tested for scenarios in which edges with increasing or decreasing length in a fully chained global tree were gradually lost/gained or replaced (Fig. 2). We tested both pure richness difference (the loss of species leading to loss of edges) and pure species replacement (keeping constant the number of species). In each modelled situation, comparisons were made between a single reference community (line on top of Fig. 2) and each subsequent community (all others below).

\section{Pure richness difference}

In the first two cases (Loss 1 and Loss 2), $\mathrm{T} \beta_{\text {total }}$ and $\mathrm{T} \beta_{\text {rich }}$ increase linearly, whereas $\mathrm{P} \beta_{\text {total }}$ or $\mathrm{F} \beta_{\text {total }}$ and $\mathrm{P} \beta_{\text {rich }}$ or $\mathrm{F} \beta_{\text {rich }}$ exhibit a curvilinear increase with increasing loss of species, depending on the direction in which the edges were lost (Fig. 3). For Loss 1, because the short edges of the tree are removed first, $\mathrm{P} \beta_{\text {total }}$ or $\mathrm{F} \beta_{\text {total }}$ and $\mathrm{P} \beta_{\text {rich }}$ or $\mathrm{F} \beta_{\text {rich }}$ increase slowly, with larger increases when the longer edges are lost. In Loss 2 , because the longer edges are lost first, $\mathrm{P} \beta_{\text {total }}$ (or F $\beta_{\text {total }}$ ) and $\mathrm{P} \beta_{\text {rich }}$ (or $\mathrm{F} \beta_{\text {rich }}$ ) increase rapidly but then level off when the removed edges decrease in length. $\beta_{\text {repl }}$ is zero in all cases.

Table 1 Matching/mismatching components $(a, b, c)$ and beta diversity values of all possible pairwise comparisons of communities in the theoretical example shown in Fig. 1.

\begin{tabular}{lllllll}
\hline \multicolumn{7}{c}{ Communities } \\
\cline { 2 - 7 } & $j, k$ & $j, l$ & $j, m$ & $k, l$ & $k, m$ & $l, m$ \\
\hline$a$ & 1 & 0 & 1 & 1 & 0.5 & 0 \\
$b$ & 0.5 & 1.5 & 0.5 & 1 & 1.5 & 1 \\
$c$ & 1 & 1 & 0 & 0 & 0.5 & 1 \\
$\beta_{\text {total }}$ & 0.6 & 1 & 0.333 & 0.5 & 0.8 & 1 \\
$\beta_{\text {repl }}$ & 0.4 & 0.8 & 0 & 0 & 0.4 & 1 \\
$\beta_{\text {rich }}$ & 0.2 & 0.2 & 0.333 & 0.5 & 0.4 & 0 \\
\hline
\end{tabular}




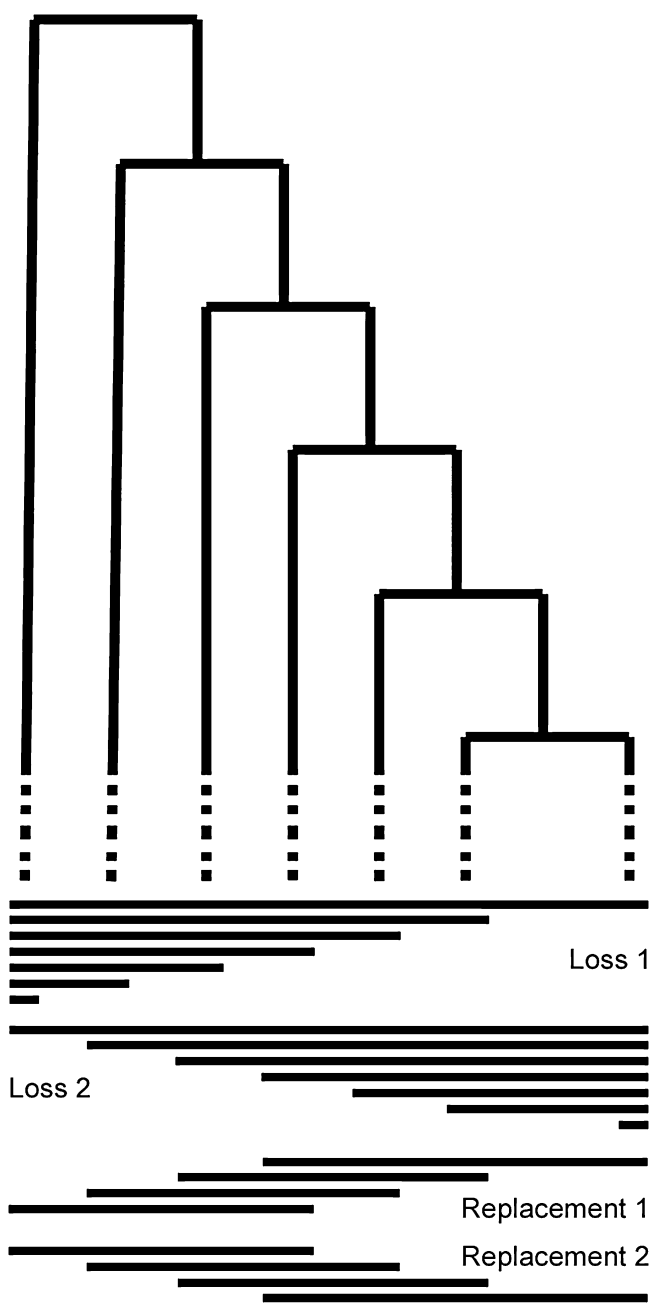

Figure 2 Theoretical example of a phylogenetic or functional tree with 100 species (to save space, only a part of the tree is shown; the tree continues to the right and down). Four examples with 100 communities each were created (thick lines show only a few of those). In relation to the first reference community (top line in each model), the first two examples show a pure loss of species, either with the short or the long edges removed first. The third and fourth scenarios represent a pure replacement of species in which species are replaced either with short or long edges removed first.

\section{Pure replacement}

The second two cases (Replacement 1 and Replacement 2) were constructed such that the number of replaced species increases monotonically and gamma diversity also increases in the same direction. As a consequence, the proportion of replaced species increases steeply at the beginning, when gamma is low (Fig. 3). This is reflected in both $\mathrm{T} \beta_{\text {total }}$ and $\mathrm{T} \beta_{\text {repl }}$ T $\mathrm{T} \beta_{\text {rich }}$ remains at zero. When applied to the phylogenetic or functional tree, the progressive species replacements lead to radically different results. In Replacement 1 , the tree of the reference community includes the shorter edges; at first, these edges are mostly replaced, and $\mathrm{P} \beta_{\text {rich }}$ (or $\mathrm{F} \beta_{\text {rich }}$ ) increases rapidly as the newly added edges represent a large proportion of gamma. $\mathrm{P} \beta_{\text {rich }}$ (or $\mathrm{F} \beta_{\text {rich }}$ ) later stabilizes, as the added edges represent a smaller proportion from the increasing gamma diversity. $\mathrm{P} \beta_{\text {repl }}$ (or $\mathrm{F} \beta_{\text {repl) }}$ increases monotonically and becomes similar to $\mathrm{P} \beta_{\text {rich }}$ (or $\mathrm{F} \beta_{\text {rich }}$ ) when replacement is complete; $\mathrm{P} \beta_{\text {total }}$ (or $\mathrm{F} \beta_{\text {total }}$ ) follows the same trend as $\mathrm{T} \beta_{\text {total }}$. In Replacement 2, the tree of the reference community includes only long edges; these are replaced first, and $\mathrm{P} \beta_{\text {repl }}$ (or $\mathrm{F} \beta_{\text {repl }}$ ) increases rapidly at the beginning. $\mathrm{P} \beta_{\text {repl }}$ (or $\mathrm{F} \beta_{\text {repl }}$ ) later stabilizes, as shorter edges are replaced. $\mathrm{P} \beta_{\text {rich }}$ (or $\mathrm{F} \beta_{\text {rich }}$ ) increases monotonically and becomes similar to $\mathrm{P} \beta$ repl (or $\mathrm{F} \beta_{\text {repl }}$ ); $\mathrm{P} \beta_{\text {total }}$ (or $\mathrm{F} \beta_{\text {total }}$ ) follows the same trend as $\mathrm{T} \beta_{\text {total }}$.

\section{Benchmark testing}

All diversity measures are capable of producing Type I and II errors when tested for statistical significance. Measures prone to Type I errors will often identify communities as deviating from randomness even when this is not the case. Measures prone to Type II errors will often not identify true deviations from randomness. To assess the robustness of the new measures to Type I and Type II errors, we performed a 'noise test' (Gotelli et al., 1997; Gotelli, 2000). We used data matrices with two assemblages and 100 species. When testing the detection of richness difference, the first assemblage contained 100 species, and the second contained zero species. When testing the detection of replacement, both assemblages contained 50 species, none of which were shared. Starting from these data and using both a star phylogeny (to test the indices when applied to taxon diversity - TD) and the dendrogram described in Fig. 2 (to test the indices when applied to phylogenetic or functional diversity - PD or FD), the species incidences were progressively interchanged, with one random change between presence and absence from each assemblage at each step with replacement (so that some species incidences may change multiple times, whereas others never change). Changes were independent for each assemblage in the pair. One hundred noise steps (added random variation) were performed, and the process was repeated 1000 times to obtain average values. In order to obtain a $P$-value for each beta measure at each noise step, the averaged observed beta values were compared to a distribution of random beta values obtained from 1000 random assemblage pairs, where each species had a $50 \%$ chance of being present in each assemblage. The proportion of $\beta$ values at each noise step that could potentially be regarded as resulting from random communities was calculated as the $P$-value of this test (probability of Type I error if the null hypothesis of a random process is rejected). Ideally, a measure would remain significantly different from random with low levels of noise and would not differ from random with high levels of noise, with a sharp turning point at intermediate levels (Gotelli, 2000). The measure is prone to Type I errors if it indicates the existence of a pattern at high levels of noise and to Type II errors if it requires little noise to obscure the pattern.

The pure loss process is undetected after approximately 20 interchanges in the case of $\beta_{\text {total }}$ and after approximately 50 

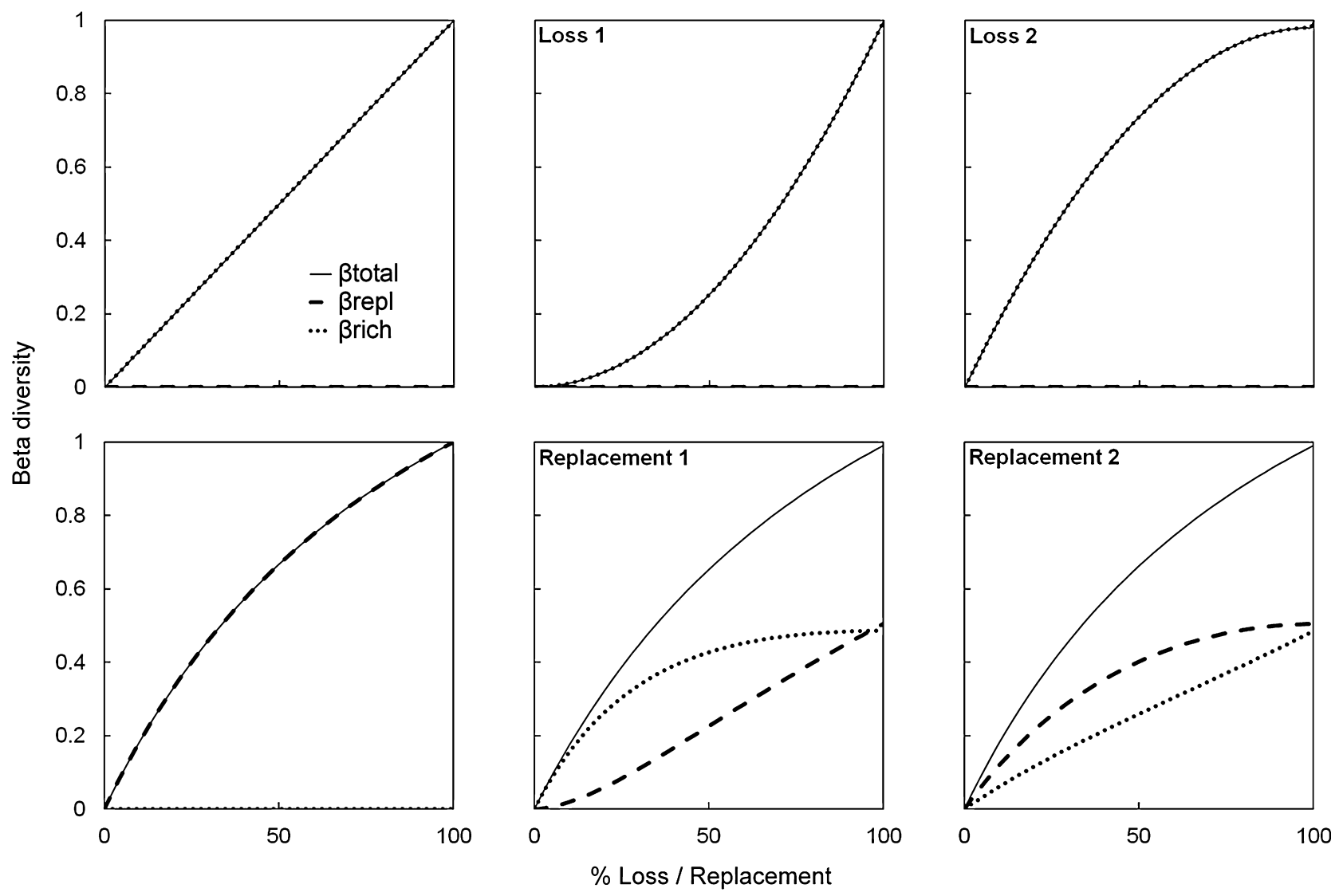

Figure 3 Results of model studies shown in Fig. 2. Top row, loss models; bottom row, replacement models. The left panels show changes in taxon beta diversity $(\mathrm{T} \beta)$; the middle and right panels show changes in phylogenetic beta diversity $(\mathrm{P} \beta)$ and functional beta diversity $(\mathrm{F} \beta)$, as measured with the trees and scenarios in Fig. 2. $\beta_{\text {total }}$, total beta diversity; $\beta_{\text {repl }}$, beta diversity due to replacement; $\beta_{\text {rich }}$, beta diversity due to richness difference.

to 70 interchanges in the case of $\beta_{\text {repl }}$ and $\beta_{\text {rich }}$ for both taxon and phylogenetic/functional diversity (Fig. 4). As random assemblage pairs have relatively similar species richness and high replacement, and because the starting point is opposite to this, the measures are very robust to high random variation in the datasets, yet do not differ from randomness when they are in fact mostly random.

The pure replacement process for taxon diversity is undetected after approximately 20 interchanges in the case of $\beta_{\text {total }}$ and $\beta_{\text {repl }}$. Because random assemblage pairs present similar richness, observed $\beta_{\text {rich }}$ (starting with 0 ) never differs from random, as expected for this particular test. For phylogenetic/functional diversity, and given the topology of the tree, $\beta_{\text {repl }}$ never differs from the random expectation. $\beta_{\text {total }}$ and $\beta_{\text {rich }}$ lose significance after approximately 20 to 30 interchanges (Fig. 4). All measures reflect randomness when assemblages are mostly random.

\section{EMPIRICAL CASES}

We use two empirical examples to demonstrate that, in many practical cases, (1) measuring $\mathrm{P} \beta$ and $\mathrm{F} \beta$ may provide insights that simple $\mathrm{T} \beta$ masks, and (2) it is fundamental to distinguish between the $\beta_{\text {repl }}$ and $\beta_{\text {rich }}$ components of $\beta_{\text {total }}$ if the observed patterns are to be fully understood and their causes are to be correctly established. Additionally, a small dataset is used to compare the new framework with previous approaches (Baselga, 2010, 2012; Leprieur et al., 2012; Villéger et al., 2013; see Appendix S1 in Supporting Information). All the statistical analyses were implemented within the $\mathrm{R}$ programming environment ( $\mathrm{R}$ Development Core Team, 2010).

\section{Phylogenetic diversity on a large spatial scale}

The Atlas of European Mammals (Mitchell-Jones et al., 1999) provides the distribution of the 160 native mammals in Europe in a $50 \mathrm{~km} \times 50 \mathrm{~km}$ resolution. We built the phylogenetic tree for these species by extracting phylogenetic relationships from the world-wide mammal supertree provided by Bininda-Emonds et al. (2007). We first downloaded the tree from http://www.evoio.org/wiki/File:Bininda-emonds_ 2007_mammals.nex (downloaded 1 March 2013). We then pruned the tree by keeping only tips that matched the species list of European mammals. Tree manipulations were achieved using the APE R package (Paradis et al., 2004). We 

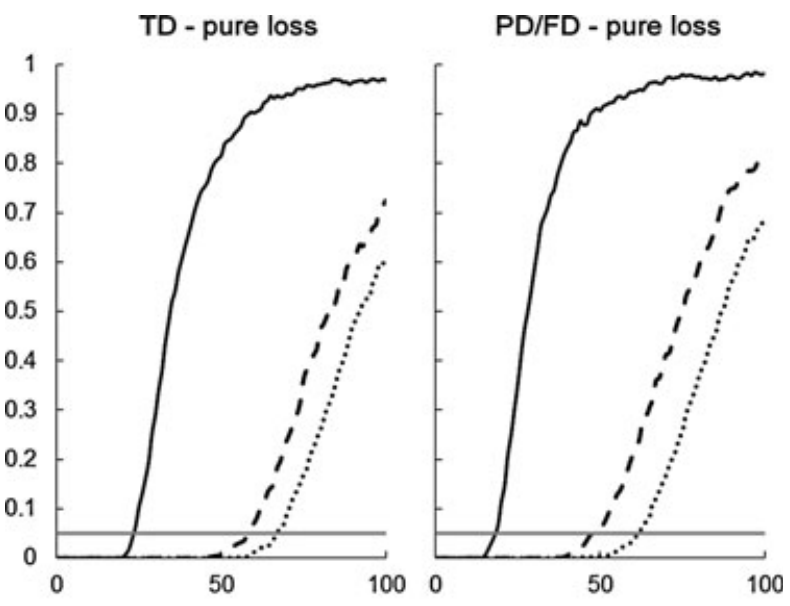

TD - pure replacement

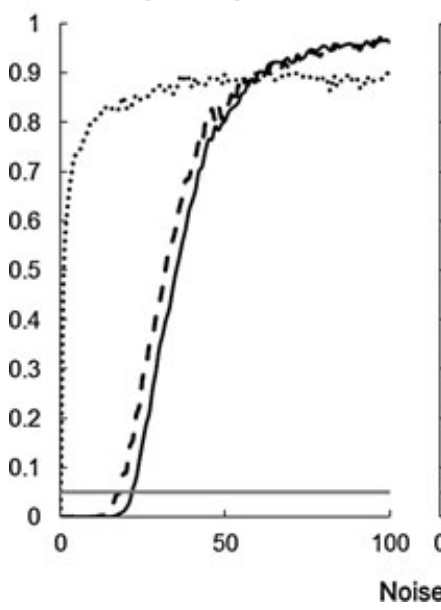

PD/FD - pure replacement

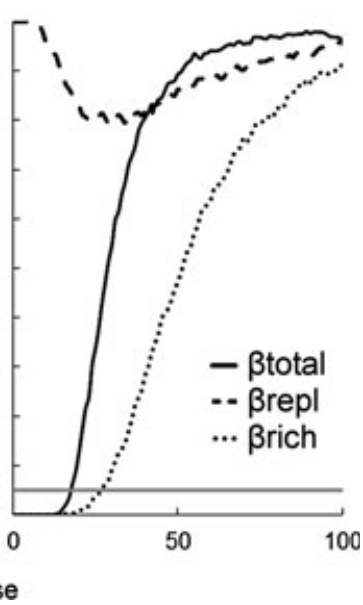

Figure 4 Benchmark testing of the new measures (TD, taxon diversity; $\mathrm{PD} / \mathrm{FD}$, phylogenetic and functional diversity). Starting with datasets with maximum richness difference (upper panels) or replacement (lower panels), the presence of species in the assemblages was progressively randomized (the $x$-axis represents noise added). The $y$-axis represents the mean $P$-value over the 1000 iterations of each benchmark testing scenario, i.e. the probability of error if the null hypothesis of a random process is rejected. In all cases, horizontal grey lines represent $P=0.05$. $\beta_{\text {total }}$, total beta diversity; $\beta_{\text {repl }}$, beta diversity due to replacement; $\beta_{\text {rich }}$, beta diversity due to richness difference.

then calculated matrices among all cells using the taxon and phylogenetic beta diversity measures as entries. We performed hierarchical agglomerative clustering of grid cells for each matrix separately, using the Ward method. A penalty function (Kelley-Gardner-Sutcliffe Kelley et al., 1996) was used to identify distinct clusters of cells. This method's minimum value corresponds to the optimal number of clusters, which is intended to represent biogeographical regions. Additionally, we calculated $\mathrm{T} \alpha$ and $\mathrm{P} \alpha$ for each cell.

$\mathrm{T} \alpha$ and $\mathrm{P} \alpha$ follow similar trends, with higher values at intermediate latitudes (Fig. 5). The use of $\mathrm{T} \beta_{\text {total }}$ and $\mathrm{P} \beta_{\text {total }}$ each identifies five clusters. Although the taxon data separate western and eastern Mediterranean areas, phylogenetic data result in a single Mediterranean cluster. Areas of low T $\alpha$ and
$\mathrm{P} \alpha$ are also clustered together (e.g. parts of Scandinavia with the Mediterranean), reflecting the influence of $\alpha$ in $\beta_{\text {total }}$. The analysis of $\mathrm{T} \beta_{\text {repl }}$ and $\mathrm{P} \beta_{\text {repl }}$ leads again to five clusters, but the pattern is clearer, with northern and central European regions and then a roughly western, central and eastern division of the Mediterranean, each extending further north than the true Mediterranean region. Taxon and phylogenetic data differ, with southern France, Greece and Norway belonging to different clusters. $\mathrm{T} \beta_{\text {rich }}$ and $\mathrm{P} \beta_{\text {rich }}$ both follow the trend of $\alpha$-diversity as expected, with the identification of four clusters.

\section{Functional diversity on a small spatial scale}

The North Atlantic Azorean archipelago, with nine islands, presents a mosaic of land uses that replaced the once almost homogeneous cover of laurel forest (Cardoso et al., 2013). A total of 72 sites arranged along a continuous gradient of disturbance according to land use and the surrounding habitat matrix in Terceira Island were sampled for epigean arthropods. The collected species were classified as endemic, native non-endemic or exotic (Cardoso et al., 2013). Functional characteristics related to resource use were collated for all the arthropod species and a functional tree was built (see Appendix S2). Based on the distribution of 32 endemic, 76 native non-endemic and 129 exotic species and their respective functional relationships in the global functional dendrogram, we then calculated taxon and functional beta-diversity matrices for all communities. We performed regressions with disturbance value differences between all possible pairs of sites as the explanatory variables and all beta diversity measures as response variables, and then tested significance with Mantel tests.

In all cases, $T \beta_{\text {total }}$ and $F \beta_{\text {total }}$ significantly increase with increasing differences in habitat disturbance (Fig. 6, Appendix S2), but for endemics the patterns are caused by increasing $\mathrm{T} \beta_{\text {rich }}$ and $\mathrm{F} \beta_{\text {rich }}$, reflecting a loss (or gain) of endemic species richness and their functions with changing disturbance. On the contrary, for native non-endemic species, the patterns follow $\mathrm{T} \beta_{\text {repl }}$ and $\mathrm{F} \beta_{\text {repl }}$, reflecting a substitution of some species by others performing different functions. The patterns for exotic species are different depending on the use of taxon or functional data. Whereas $\mathrm{T} \beta_{\text {rich }}$ causes the pattern of taxon beta, $F \beta_{\text {repl }}$ causes the functional pattern. This difference reflects a change of exotic species richness along the gradient of disturbance but mostly a replacement of functions along the same gradient.

\section{DISCUSSION}

Using a range of both theoretical and empirical examples, we confirm that taxon data - i.e. species identities alone - do not allow the recovery of patterns and processes that require information on how species are phylogenetically related and/ or how they exploit and share resources. The unified framework used here combines taxon, phylogenetic and functional 

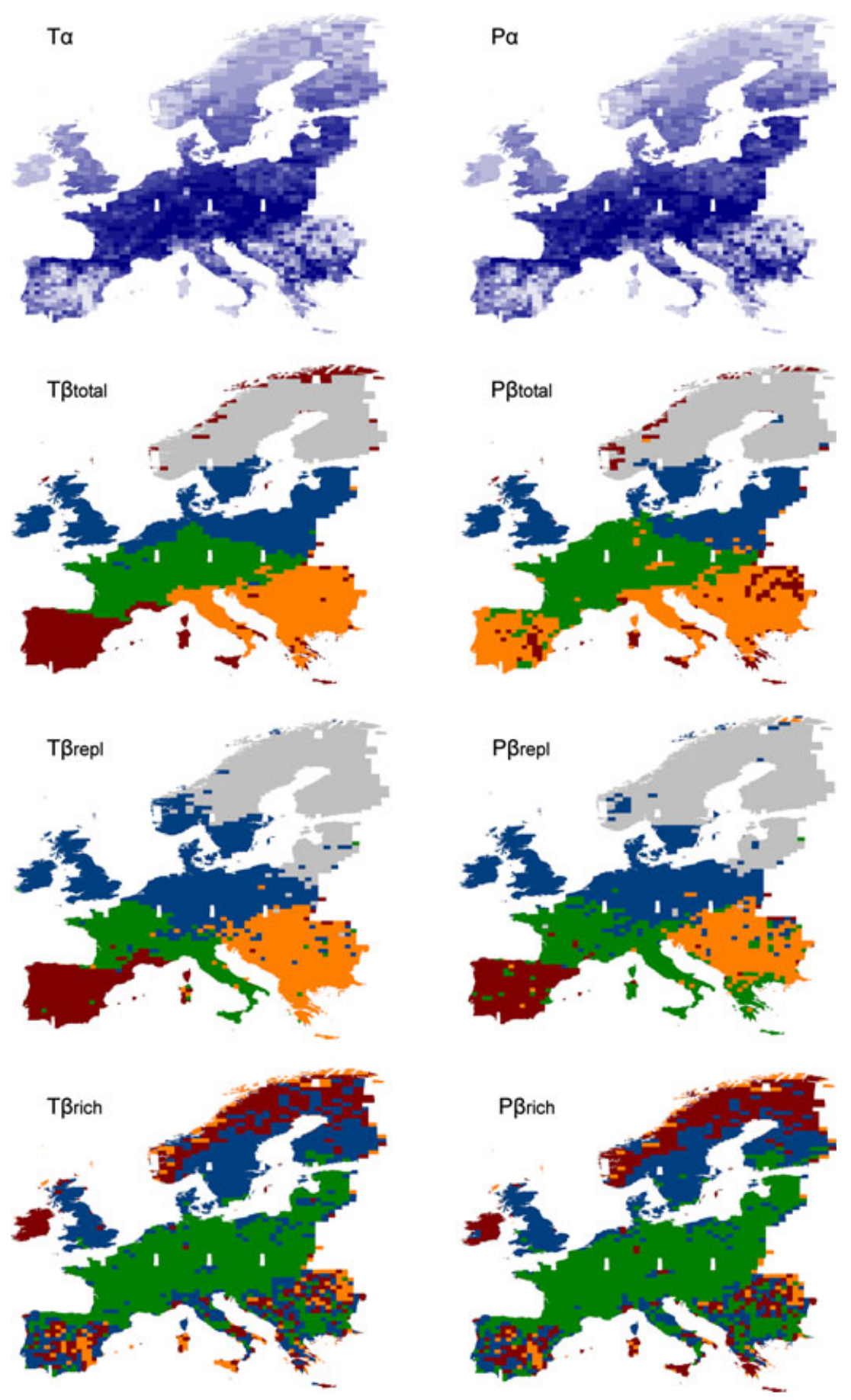

Figure 5 Geographical distribution of clusters derived from taxon beta diversity $(\mathrm{T} \beta)$ and phylogenetic beta diversity $(\mathrm{P} \beta)$ data of European mammals. Different colours/shades represent different clusters. Maps of $\alpha$-diversity are shown for comparison, with darker shades representing higher values. Owing to coordinate mismatch, interspersed blank areas have no data. $\beta_{\text {total }}$, total beta diversity; $\beta_{\text {repl }}$, beta diversity due to replacement; $\beta_{\text {rich }}$, beta diversity due to richness difference.

diversity into a single tree-based approach, offering meaningful comparisons between these facets of biological diversity using the same set of community data.

We demonstrate that 'catch-all' beta diversity measures, such as UniFrac or PhyloSor, do not allow differences driven by the replacement of lineages or functional traits to be distinguished from those driven by the loss (or gain) of entire lineages or traits. Our approach to diversity partitioning differs from the methods suggested by Baselga (2010, 2012), Leprieur et al. (2012) and Villéger et al. (2013) in that pairwise dissimilarities between communities are explicitly separated into a component from replacement and another corresponding to richness difference. The previous approaches consistently overestimate the part of beta diversity that derives from true replacement and underestimate the part that derives from richness difference, as previously shown for TD (see also Appendix S1); further advantages are detailed elsewhere (Schmera \& Podani, 2011; Carvalho et al., 2012, 2013).

The different measures also do not appear to be prone to either Type I or Type II errors. They are able to detect patterns with moderate to high amounts of random variation in 


\section{Taxon diversity}
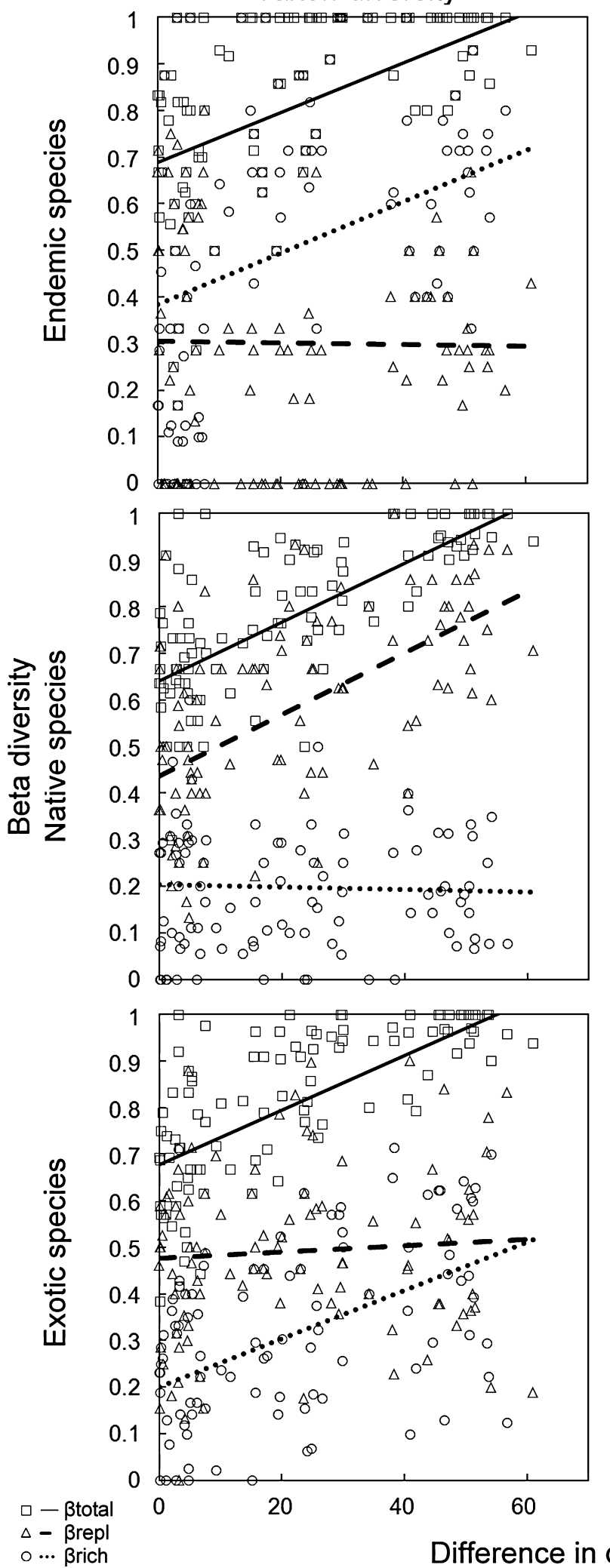

Functional diversity
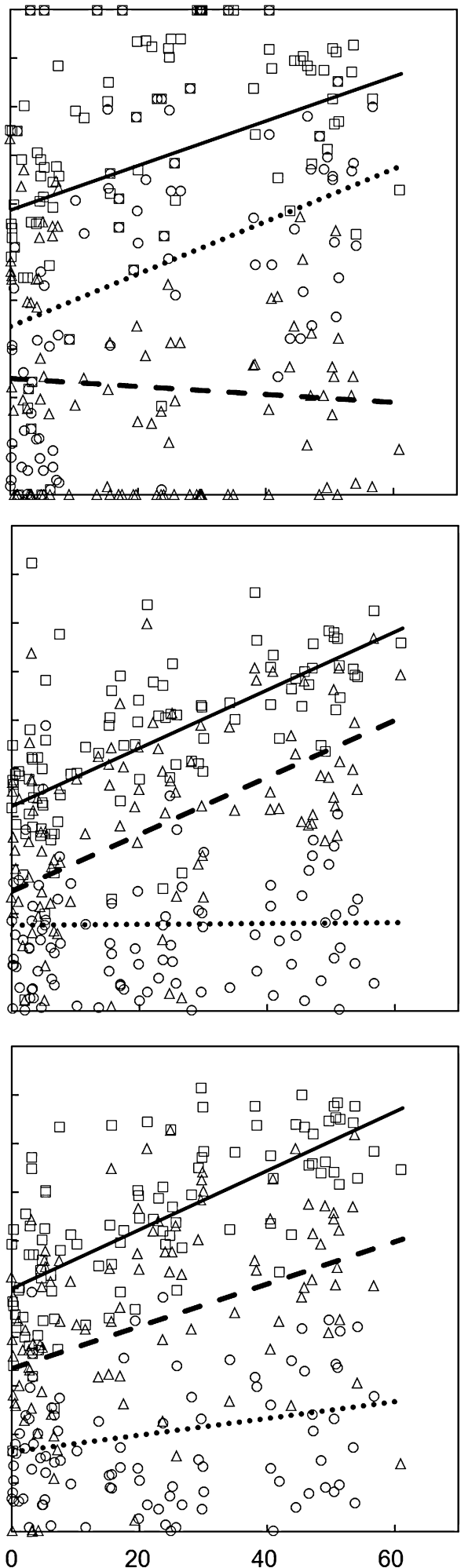

Figure 6 Linear regressions of differences in disturbance index and beta diversity among all possible pairs of 72 sites in Terceira Island (Azores) that were sampled for arthropods. Only 100 dots per regression are shown for clarity. $\beta_{\text {total }}$, total beta diversity; $\beta_{\text {repl }}$, beta diversity due to replacement; $\beta_{\text {rich }}$, beta diversity due to richness difference. See Appendix S2 for the slope, $r^{2}$ and significance values. 
the data (have good statistical power) and the measures do not differ from random values when communities are indeed mostly random (low Type I error rate). In the pure replacement scenario, $\beta_{\text {rich }}$ of taxon diversity and $\beta_{\text {repl }}$ for phylogenetic and functional diversity were never significant (different from random values) simply because the testing scenario did not alter richness or the phylogeny or functional characteristics of the species.

\section{Phylogenetic diversity of European mammals}

Depending on the diversity measure used, our clusters either do or do not match those previously obtained by Heikinheimo et al. (2007). During the analysis of total beta diversity, one or two large southern European clusters appear, with south-western and south-eastern Europe being differentiated when no phylogenetic information is taken into account. The analysis of $\beta_{\text {repl }}$ alone presents a different perspective from this and from Heikinheimo et al. (2007), with southern Europe being divided into three areas, roughly corresponding to the Mediterranean peninsulas. It appears that richness differences were confounding the species-level turnover between regions of southern Europe. These areas acted as glacial refugia and are often thought to harbour greatly different faunas, although they are currently depauperate compared with central Europe. However, a similar level and pattern of provinciality have been characteristic of the European mammal fauna for millions of years (Maridet et al., 2007; Costeur \& Legendre, 2008). In the fossil data, the degree to which the peninsulas are separate is strongly dependent on the taxonomic level analysed: genus-level analyses yield a much weaker pattern of endemism than species-level analyses. The high replacement of species and lineages between the peninsulas observed in our analysis may similarly be due to the presence of local species-level endemics. This indicates that, although the Iberian Peninsula differs in terms of species composition, this region shares a common phylogenetic history with other Mediterranean areas.

Richness differences should, in principle, be higher in areas with stronger environmental gradients, when extremes of cold or drought are reached. Such extremes could cause the fine-scale variation of $\beta_{\text {rich }}$ within the Iberian and Balkan peninsulas, with a marked loss of species in particularly arid ecosystems (Casanovas-Vilar \& Agustí, 2007). Additionally, the fossil record reveals a pattern of basin-level endemism in the Iberian Peninsula, especially for small non-volant mammals (Agustí, 1990).

The reasons for separation of the Mediterranean peninsulas from the continental clusters differ from one another. For Iberia, the main factor is undoubtedly geographical isolation with consequent endemism (van der Meulen et al., 2011). For Italy, the situation is somewhat similar today, although its geological history is much more complicated (Rook et al., 2006). For the Balkans, the background is different in that this area forms the westernmost extension of many Asian biotas, which, when Asia is excluded from the analysis, causes the region to appear highly 'endemic' (Maridet et al., 2007; Costeur \& Legendre, 2008).

\section{Functional diversity of Azorean arthropods}

The similar increase in $T \beta_{\text {total }}$ and $F \beta_{\text {total }}$ with increasing habitat disturbance differences for all species groups reveals the presence of species adapted to each extreme of the gradient and the respective functional change. However, partitioning $\mathrm{T} \beta_{\text {total }}$ and $\mathrm{F} \beta_{\text {total }}$ into their respective $\beta_{\text {repl }}$ and $\beta_{\text {rich }}$ components reveals that the increases of $T \beta_{\text {total }}$ and $F \beta_{\text {total }}$ were not driven by the same processes for the different communities. For endemics, $T \beta_{\text {total }}$ and $F \beta_{\text {total }}$ are simply an outcome of $\mathrm{T} \beta_{\text {rich }}$ and $\mathrm{F} \beta_{\text {rich }}$, reflecting the decline of both species and the range of functions they perform along the disturbance gradient. Endemic species are expected to be more sensitive to habitat disturbance, and only a few of them are able to sustain populations outside native forests (Cardoso et al., 2013). The relative stability of $\mathrm{T} \beta_{\text {repl }}$ and $\mathrm{F} \beta_{\text {repl }}$ along the gradient indicates that increasing disturbance differences does not increase true taxon and functional turnover between communities. That is, species and functions are replaced to the same degree when comparing similar or very different communities. This last point may reflect the fact that endemics often have very restricted distributions and that even among pristine habitats, the species performing similar functions are replaced. This replacement among seemingly similar habitats is as strong as the replacement of species and traits from pristine to highly disturbed habitats, as only a few endemics are present in the latter. By contrast, $\mathrm{T} \beta_{\text {total }}$ and $\mathrm{F} \beta_{\text {total }}$ of native non-endemic species are mainly driven by $\mathrm{T} \beta_{\text {repl }}$ and $\mathrm{F} \beta_{\text {repl. }}$. This indicates that native species richness is less affected by disturbance intensification and that the replacement of species is coupled with a corresponding replacement of functions. Native non-endemic species communities in highly disturbed habitats are taxonomically and functionally distinct from those occurring in little-disturbed habitats, but both hold similar species richness and a similar range of functions. Contrary to endemic and native nonendemic species, the increase of $T \beta_{\text {total }}$ and $F \beta_{\text {total }}$ differences with increasing disturbance differences for exotic species is not explained by the same processes. The pattern of $\mathrm{T} \beta_{\text {total }}$ is mainly driven by $\mathrm{T} \beta_{\text {rich }}$, reflecting the gain of exotic species when disturbance increases (Cardoso et al., 2013). T $\beta_{\text {repl }}$ is constant but greater than zero along the gradient, also revealing an important contribution of the turnover of species to total differentiation, although it tends to retain importance along the gradient. Opposite to this pattern, $F \beta_{\text {repl }}$ - reflecting the replacement of functions along the gradient - mostly causes the pattern in $\mathrm{F} \beta_{\text {total }}$. These findings indicate that less exotic species occur in habitats with low disturbance compared to highly disturbed habitats, but this increase in the number of species is mostly reflected by a replacement of functional traits. With increasing disturbance, many more exotic species encompass only a slightly larger range of traits, even if such traits are completely different along the gradient. 


\section{Future perspectives}

Comparing TD, PD and FD requires a common framework. Using the methodology proposed here, these three 'dimensions' are fully comparable, because identical measures are applied to all of them. Although we have used trees as a means to guarantee direct comparability of the different dimensions, this framework can be easily adapted to other representations. For example, it is common for functional diversity to be represented in a multidimensional volume, in which case beta diversity and the matching/mismatching components are quantified according to the degree of overlap between the volumes that are occupied by the different communities (Villéger et al., 2013). Additionally, as presented here, the methods consider species presence/absence only, but extension to abundances is possible using a weighting scheme (Cadotte et al., 2010).

Our two empirical examples confirm that comparing $\mathrm{P} \beta$ and $\mathrm{F} \beta$ with the most commonly used $\mathrm{T} \beta$ reveals previously hidden patterns of beta diversity. For instance, a predominant increase in $\mathrm{P} \beta_{\text {repl }}$ or $\mathrm{F} \beta_{\text {repl }}$ along a gradient without an equivalent increase in $\mathrm{T} \beta_{\text {repl }}$ may reflect historical factors that cause entire lineages to be represented in part of the studied area only, either because they are replaced by completely different lineages elsewhere or because environmental factors force the occurrence of different traits in different communities. By contrast, a predominant increase of $\mathrm{P} \beta_{\text {rich }}$ may reflect dispersal limitation for certain lineages. For example, the inability of some high-level taxa to reach isolated islands is known, causing the disharmony of many oceanic islands (Whittaker \& Fernández-Palacios, 2007), probably causing higher $\mathrm{P} \beta_{\text {rich }}$ than expected by the corresponding $\mathrm{T} \beta_{\text {rich }}$ values. It may also reflect environmental filtering, which dictates that only species with given traits are able to occupy certain habitats. For example, caves are occupied by taxa that have pre-adaptations to the particular biotic and abiotic conditions that exist in the subterranean realm (Culver \& Pipan, 2009), probably causing higher $F \beta_{\text {rich }}$ than expected from the corresponding $\mathrm{T} \beta_{\text {rich }}$ values.

We also demonstrate that partitioning $\mathrm{P} \beta_{\text {total }}$ and $\mathrm{F} \beta_{\text {total }}$ into $\beta_{\text {repl }}$ and $\beta_{\text {rich }}$ components reveals more complex patterns than using the overall coefficients alone, further elucidating the different forces operating in community assembly. For instance, if a phylogenetic pattern is dominated by $\beta_{\text {repl }}$, there is a strong replacement of lineages from community to community without a notable difference in the amount of phylogenetic information encompassed by the different communities. This may occur in islands with similar areas and ages within the same archipelago, each with its own unique radiations. On the contrary, a phylogenetic pattern dominated by $\beta_{\text {rich }}$ may occur in islands with very different areas and/or ages, in which smaller or younger islands present subsets of the species present in larger or older islands, as limited area, time or niche pre-emption may prevent an extensive radiation of lineages (Silvertown et al., 2005). A functional pattern dominated by $\beta_{\text {repl }}$ may occur in habitats that require very different traits but with similar richness. For example, we refer to two different types of forests comprising different tree species but with similar richness of niches for herbivores. By contrast, a functional pattern that is dominated by $\beta_{\text {rich }}$ may reflect environmental filtering that favours certain traits over others along a gradient (Kluge \& Kessler, 2011).

\section{ACKNOWLEDGMENTS}

We thank Nick Gotelli, Joe Veech and David Nipperess for comments on previous versions of the manuscript. Clara Gaspar compiled all the functional data on Azorean arthropods. The Societas Europaea Mammalogica and particularly Tony Mitchell-Jones provided the European Mammals Atlas data. Isaac Casanovas-Vilar and Raymond L. Bernor enlightened us regarding Iberian endemism in the fossil record. Azorean data derive from the DRCT project M.2.1.2/I/ $003 / 2008$. F.R. and P.B. were partly supported by FCT project PTDC/BIA-BIC/119255/2010. J.P. and D.S. were supported by the Hungarian Scientific Research Fund (OTKA K104279).

\section{REFERENCES}

Agustí, J. (1990) The Miocene rodent succession in eastern Spain: a zoogeographical appraisal. European Neogene mammal chronology (ed. by E.H. Lindsay, V. Fahlbusch and P. Mein), pp. 375-404. Plenum Press, New York.

Almeida-Neto, M., Frensel, D.M.B. \& Ulrich, W. (2012) Rethinking the relationship between nestedness and beta diversity: a comment on Baselga (2011). Global Ecology and Biogeography, 21, 772-777.

Anderson, M.J., Crist, T.O., Chase, J.M., Vellend, M., Inouye, B.D., Freestone, A.L., Sanders, N.J., Cornell, H.V., Comita, L.S., Davies, K.F., Harrison, S.P., Kraft, N.J.B., Stegen, J.C. \& Swenson, N.G. (2011) Navigating the multiple meanings of $\beta$ diversity: a roadmap for the practicing ecologist. Ecology Letters, 14, 19-28.

Baiser, B., Olden, J.D., Record, S., Lockwood, J.L. \& McKinney, M.L. (2012) Pattern and process of biotic homogenization in the New Pangaea. Proceedings of the Royal Society B: Biological Sciences, 279, 4772-4777.

Baselga, A. (2010) Partitioning the turnover and nestedness components of beta diversity. Global Ecology and Biogeography, 19, 134-143.

Baselga, A. (2012) The relationship between species replacement, dissimilarity derived from nestedness, and nestedness. Global Ecology and Biogeography, 21, 1223-1232.

Bininda-Emonds, O.R.P., Cardillo, M., Jones, K.E., MacPhee, R.D.E., Beck, R.M.D., Grenyer, R., Price, S.A., Vos, R.A., Gittleman, J.L. \& Purvis, A. (2007) The delayed rise of present-day mammals. Nature, 446, 507-512.

Bryant, J.A., Lamanna, C., Morlon, H., Kerkhoff, A.J., Enquist, B.J. \& Green, J.L. (2008) Microbes on mountainsides: contrasting elevational patterns of bacterial and 
plant diversity. Proceedings of the National Academy of Sciences USA, 105, 11505-11511.

Cadotte, M.W., Davies, T.J., Regetz, J., Kembel, S.W., Cleland, E. \& Oakley, T.H. (2010) Phylogenetic diversity metrics for ecological communities: integrating species richness, abundance and evolutionary history. Ecology Letters, 13, 96-105.

Cardoso, P., Borges, P.A.V. \& Veech, J.A. (2009) Testing the performance of beta diversity measures based on incidence data: the robustness to undersampling. Diversity and Distributions, 15, 1081-1090.

Cardoso, P., Rigal, F., Fattorini, S., Terzopoulou, S. \& Borges, P.A.V. (2013) Integrating landscape disturbance and indicator species in conservation studies. PLOS ONE, 8, e63294.

Carvalho, J.C., Cardoso, P. \& Gomes, P. (2012) Determining the relative roles of species replacement and species richness differences in generating beta-diversity patterns. Global Ecology and Biogeography, 21, 760-771.

Carvalho, J.C., Cardoso, P., Borges, P.A.V., Schmera, D. \& Podani, J. (2013) Measuring fractions of beta diversity and their relationships to nestedness: a theoretical and empirical comparison of novel approaches. Oikos, 122, 825-834.

Casanovas-Vilar, I. \& Agustí, J. (2007) Ecogeographical stability and climate forcing in the Late Miocene (Vallesian) rodent record of Spain. Palaeogeography, Palaeoclimatology, Palaeoecology, 248, 169-189.

Chao, A., Chiu, C.-H. \& Jost, L. (2010) Phylogenetic diversity measures based on Hill numbers. Philosophical Transactions of the Royal Society B: Biological Sciences, 365, 3599-3609.

Costeur, L. \& Legendre, S. (2008) Spatial and temporal variation in European Neogene large mammals diversity. Palaeogeography, Palaeoclimatology, Palaeoecology, 261, 127-144.

Culver, D.C. \& Pipan, T. (2009) Biology of caves and other subterranean habitats. Oxford University Press, Oxford.

Devictor, V., Mouillot, D., Meynard, C., Jiguet, F., Thuiller, W. \& Mouquet, N. (2010) Spatial mismatch and congruence between taxonomic, phylogenetic and functional diversity: the need for integrative conservation strategies in a changing world. Ecology Letters, 13, 1030-1040.

Faith, D.P. (1992) Conservation evaluation and phylogenetic diversity. Biological Conservation, 61, 1-10.

Feng, G., Zhang, J.-L., Pei, N.-C., Rao, M.-D., Mi, X.-C., Ren, H.-B. \& Ma, K.-P. (2012) Comparison of phylobetadiversity indices based on community data from Gutianshan forest plot. Chinese Science Bulletin, 57, 623-630.

Gotelli, N.J. (2000) Null model analysis of species co-occurrence patterns. Ecology, 81, 2606-2621.

Gotelli, N.J., Buckley, N.J. \& Wiens, J.A. (1997) Co-occurrence of Australian land birds: Diamond's assembly rules revisited. Oikos, 80, 311-324.

Graham, C. \& Fine, P. (2008) Phylogenetic beta diversity: linking ecological and evolutionary processes across space and time. Ecology Letters, 11, 1265-1277.
Heikinheimo, H., Fortelius, M., Eronen, J. \& Mannila, H. (2007) Biogeography of European land mammals shows environmentally distinct and spatially coherent clusters. Journal of Biogeography, 34, 1053-1064.

Helmus, M.R., Bland, T.J., Williams, C.K. \& Ives, A.R. (2007) Phylogenetic measures of biodiversity. The American Naturalist, 169, E68-E83.

Jost, L. (2007) Partitioning diversity into independent alpha and beta components. Ecology, 88, 2427-2439.

Kelley, L.A., Gardner, S.P. \& Sutcliffe, M.J. (1996) An automated approach for clustering an ensemble of NMRderived protein structures into conformationally related subfamilies. Protein Engineering, 9, 1063-1065.

Kluge, J. \& Kessler, M. (2011) Phylogenetic diversity, trait diversity and niches: species assembly of ferns along a tropical elevational gradient. Journal of Biogeography, 38, 394-405.

Lande, R. (1996) Statistics and partitioning of species diversity, and similarity among multiple communities. Oikos, 76, 5-13.

Legendre, P. \& Legendre, L. (2012) Numerical ecology, 3rd edn. Elsevier, Amsterdam.

Lennon, J.J., Koleff, P., Greenwood, J.J.D. \& Gaston, K.J. (2001) The geographical structure of British bird distributions: diversity, spatial turnover and scale. Journal of Animal Ecology, 70, 966-979.

Leprieur, F., Albouy, C., De Bortoli, J., Cowman, P.F., Bellwood, D.R. \& Mouillot, D. (2012) Quantifying phylogenetic beta diversity: distinguishing between 'true' turnover of lineages and phylogenetic diversity gradients. PLoS ONE, 7, e42760.

Lozupone, C.A. \& Knight, R. (2005) UniFrac: a new phylogenetic method for comparing microbial communities. Applied Environmental Microbiology, 71, 8228-8235.

Maridet, O., Escarguel, G., Costeur, L., Mein, P., Hugueney, M. \& Legendre, S. (2007) Small mammal (rodents and lagomorphs) European biogeography from the Late Oligocene to the mid Pliocene. Global Ecology and Biogeography, 16, 529-544.

Marini, L., Bertolli, A., Bona, E., Federici, G., Martini, F., Prosser, F. \& Bommarco, R. (2013) Beta-diversity patterns elucidate mechanisms of alien plant invasion in mountains. Global Ecology and Biogeography, 22, 450-460.

van der Meulen, A.J., García-Paredes, I., Álvarez-Sierra, M.Á., van den Hoek Ostende, L.W., Hordijk, K., Oliver, A., López-Guerrero, P., Hernández-Ballarín, V. \& PeláezCampomanes, P. (2011) Biostratigraphy or biochronology? Lessons from the Early and Middle Miocene small mammal events in Europe. Geobios, 44, 309-321.

Meynard, C.N., Devictor, V., Mouillot, D., Thuiller, W., Jiguet, F. \& Mouquet, N. (2011) Beyond taxonomic diversity patterns: how do $\alpha, \beta$ and $\gamma$ components of bird functional and phylogenetic diversity respond to environmental gradients across France? Global Ecology and Biogeography, 20, 893-903.

Mitchell-Jones, A.J., Amori, G., Bogdanowicz, W., Krystufek, B., Reijnders, P.J.H., Spitzenberger, F., Stubbe, M., 
Thissen, J.B.M., Vohralík, V. \& Zima, J. (1999) The atlas of European mammals. T. \& A.D. Poyser and Academic Press, London.

Nipperess, D.A., Faith, D.P. \& Barton, K. (2010) Resemblance in phylogenetic diversity among ecological assemblages. Journal of Vegetation Science, 21, 809-820.

Paradis, E., Claude, J. \& Strimmer, K. (2004) APE: analyses of phylogenetics and evolution in $\mathrm{R}$ language. Bioinformatics, 20, 289-290.

Petchey, O.L. \& Gaston, K.J. (2002) Functional diversity (FD), species richness and community composition. Ecology Letters, 5, 402-411.

Petchey, O.L. \& Gaston, K.J. (2006) Functional diversity: back to basics and looking forward. Ecology Letters, 9, 741-758.

Podani, J. \& Schmera, D. (2011) A new conceptual and methodological framework for exploring and explaining pattern in presence-absence data. Oikos, 120, 1625-1638.

Podani, J., Ricotta, C. \& Schmera, D. (2013) A general framework for analyzing beta diversity, nestedness and related community-level phenomena based on abundance data. Ecological Complexity, 15, 52-61.

R Development Core Team (2010) R: a language and environment for statistical computing. Version 2.13.0. R Foundation for Statistical Computing, Vienna, Austria. Available at: http://www.r-project.org/.

Rao, C.R. (1982) Diversity and dissimilarity coefficients: a unified approach. Theoretical Population Biology, 21, 2443.

Ricotta, C. \& Burrascano, S. (2008) Beta diversity for functional ecology. Preslia, 80, 61-71.

Ricotta, C., Bacaro, G., Marignani, M., Godefroid, S. \& Mazzoleni, S. (2012) Computing diversity from dated phylogenies and taxonomic hierarchies: does it make a difference to the conclusions? Oecologia, 170, 501-506.

Rook, L., Gallai, G. \& Torre, D. (2006) Lands and endemic mammals in the Late Miocene of Italy: constraints for paleogeographic outlines of Tyrrhenian area. Palaeogeography, Palaeoclimatology, Palaeoecology, 238, 263-269.

Schmera, D. \& Podani, J. (2011) Comments on the separating components of beta diversity. Community Ecology, 12, 153-160.

Silvertown, J., Ortega, J.F. \& Carine, M. (2005) The monophyly of island radiations: an evaluation of niche pre-emption and some alternative explanations. Journal of Ecology, 93, 653-657.

Stegen, J.C. \& Hurlbert, A.H. (2011) Inferring eco-evolutionary processes from taxonomic, phylogenetic and functional trait $\beta$-diversity. PLoS ONE, 6, e20906.

Swenson, N.G. (2011) Phylogenetic beta diversity metrics, trait evolution and inferring the functional beta diversity of communities. PLoS ONE, 6, e21264.
Tilman, D., Reich, P.B., Knops, J., Wedin, D., Mielke, T. \& Lehman, C. (2001) Diversity and productivity in a longterm grassland experiment. Science, 294, 843-845.

Veech, J., Summerville, K., Crist, T. \& Gering, J. (2002) The additive partitioning of species diversity: recent revival of an old idea. Oikos, 99, 3-9.

Villéger, S., Miranda, J.R., Hernandez, D.F. \& Mouillot, D. (2012) Low functional $\beta$-diversity despite high taxonomic $\beta$-diversity among tropical estuarine fish communities. PLoS ONE, 7, e40679.

Villéger, S., Grenouillet, G. \& Brosse, S. (2013) Decomposing functional $\beta$-diversity reveals that low functional $\beta$-diversity is driven by low functional turnover in European fish assemblages. Global Ecology and Biogeography, 22, 671-681.

Webb, C.O., Ackerly, D.D., McPeek, M.A. \& Donoghue, M.J. (2002) Phylogenies and community ecology. Annual Review of Ecology, Evolution, and Systematics, 33, 475-505.

Whittaker, R.H. (1960) Vegetation of the Siskiyou Mountains, Oregon and California. Ecological Monographs, 30, 279-338.

Whittaker, R.J. \& Fernández-Palacios, J.M. (2007) Island biogeography: ecology, evolution, and conservation, 2nd edn. Oxford University Press, Oxford.

\section{SUPPORTING INFORMATION}

Additional Supporting Information may be found in the online version of this article:

Appendix S1 Phylogenetic and functional analysis of Christmas Island rats.

Appendix S2 Additional information on the Azorean arthropods analyses.

\section{BIOSKETCH}

Pedro Cardoso is a curator at the Finnish Museum of Natural History, University of Helsinki. His research interests include biogeography, conservation biology, biodiversity assessment, spider taxonomy and computer science, namely on the field of artificial intelligence. He is currently developing a number of novel biodiversity descriptors, statistical methodology and software packages for better knowledge of biodiversity patterns.

Author contributions: P.C., F.R. and J.C.C. conceived the ideas; F.R., M.F. and P.A.V.B. collected the data; P.C. and F.R. analysed the data; and P.C., J.P. and D.S. led the writing, with contributions from all authors.

Editor: Joseph Veech 